巽519485(1)

UCRL-ID-118631

\title{
RAS Division Model Evaluation Database Description
}

\author{
Kevin T. Foster \\ Regional Atmospheric Sciences Division \\ Lawrence Livermore National Laboratory \\ Livermore, California 94551 USA
}

September 1994

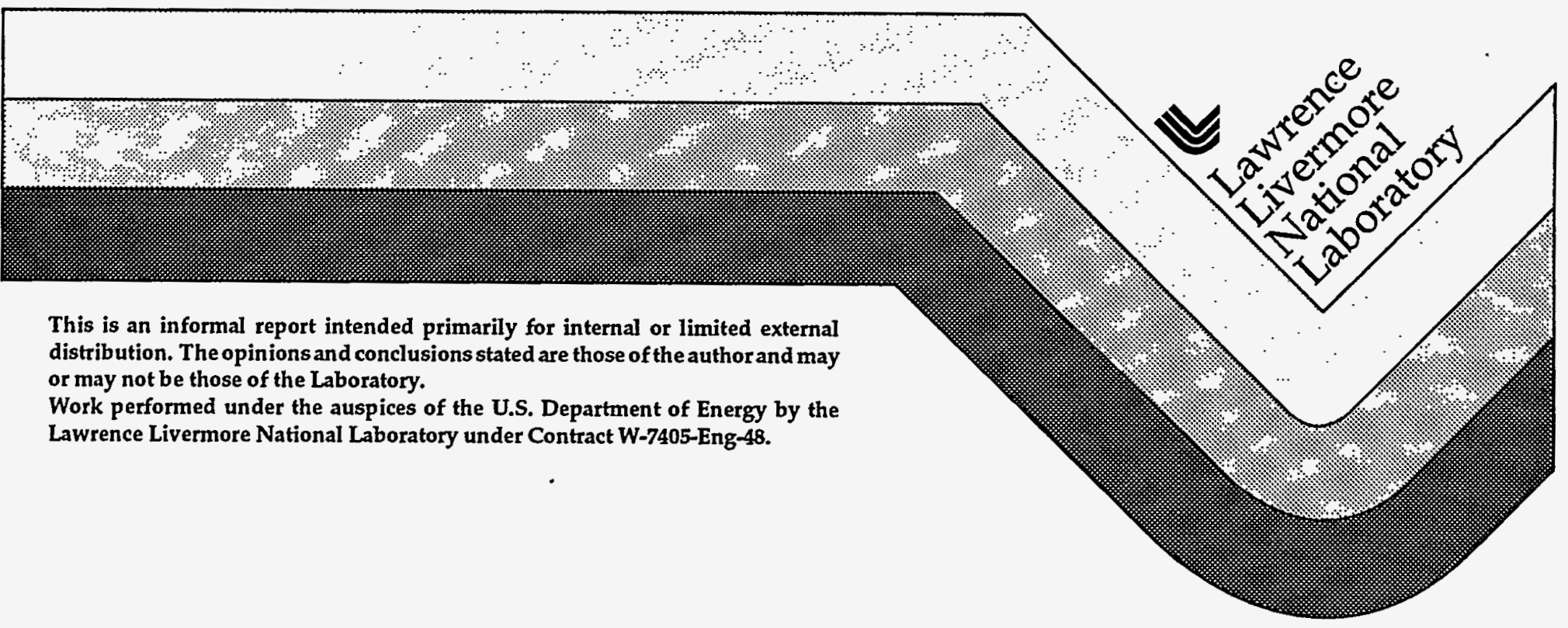




\section{DISCLAIMER}

This document was prepared as an acccount of work sponsored by an agency of the United States Government. Neither the United States Government nor the University of California nor any of their employees, makes any warranty, express or implied, or assumes any legal liability or responsibility for the accuracy, completeness, or usefulness of any information, apparatus, product, or process disclosed, or represents that its use would not infringe privately own rights. Reference herein to any specifie commercial products, process, or service by trade name, trademark, manufacturer, or otherwise, does not necessarily constitute or imply its endorsement, recommendation, or favoring by the United States Government or the University of California. The views and opinions of authors expressed herein do not necessarily state or reflect those of the United States Government or the University of California, and shall not be used for advertising or product endorsement purposes.

This report has been reproduced directly from the best available copy.

Available to DOE and DOE contractors from the Office of Scientific and Technical Information P.O. Box 62, Oak Ridge, TN 37831

Prices available from (615) 576-8401, FIS 626-8401

Available to the public from the National Technical Information Service

U.S. Department of Commerce 5285 Port Royal Rd. Springfield, VA 22161 


\section{DISCLAIMER}

Portions of this document may be illegible in electronic image products. Images are produced from the best available original document. 


\section{RAS Division \\ Model Evaluation Database \\ Description}

Kevin Foster

September 1, 1994

\section{Introduction}

Over the past several years what is now the Regional Atmospheric Sciences (RAS) Division conducted several atmospheric model evaluation studies (see for example "An Updated Summary of MATHEW/ADPIC Model Evaluation Studies", Foster and Dickerson, UCRL-JC-104134). These studies made use of numerous tracer field experiment datasets comprised of both meteorological and tracer concentration data. Over the years these datasets have often been misplaced, or otherwise lost. This obviously made it very difficult to rerun these studies for comparison with results of later, hopefully improved, versions of the RAS Division models, or to otherwise make use of these data.

Recently, planning has begun for the potential development of a set of "model evaluation" tools for the Division's use. The primary function of these tools will be to facilitate comparison of model calculations with measurements of both atmospheric variables (e.g. wind velocity, temperatures, etc.) and the atmosphere's transport and dispersion effects on the concentration of atmospheric tracers.

To supply these tools with the necessary atmospheric measurements, and to create a relatively more permanent archive of field experiment data, a Model Evaluation Database (MEDb) has been designed and implemented. This document contains a description of the contents and current structure of the MEDb', and provides guidance on determining appropriate database entries. An associated document (RAS Division Model Evaluation Database Tools) explains the existing methods to view and modify the data. The reader is cautioned that these methods, and the Database structure itself, will likely change as the model evaluation system capabilities evolve and additional requirements are defined.

Figure 1 presents a very top-level working diagram of the anticipated major components of the model evaluation system. External to these primary "domain" components is the supporting Model Data and Input Archive which is comprised of measurements, measurement station information, and tracer source data. This archive is primarily meant to provide necessary input to the model evaluation system and, secondarily, to supply a potential source of appropriate data for atmospheric model input. The MEDb has been designed to meet these functions.

This document first contains a general description of the contents of the MEDb. This is followed by a discussion of the MEDb file structure as it is currently implemented. A final section contains a discussion of issues related to filling the MEDb with data. Document appendices contain a detailed description of the $\mathrm{MEDb}$ record and file structure. 


\section{MEDb Contents}

The MEDb is divided into six groupings of information associated with each given field experiment or set of related field experiments (e.g. DOPPTEX set, MATS set, 1991 ASCOT/WVS set, etc.). All of the data generated by such related field experiments constitute a "dataset". Three of these groupings contain relatively "high-level" descriptive dataset information. This "high-level" dataset information is archived in three separate series of storage structures with the following "logical" names:

1) General Dataset Information,

2) Dataset Measurement Type Information, and

3) Dataset Source Term Information.

The remaining three "lower-level" groupings contain data related to a given Measurement Type (e.g. temperature, pressure, wind speed and direction, tracer concentration, etc.) found in a particular dataset. This information is also archived in three series of separate storage structures which have these "logical" names:

1) Measurement Station Information,

2) Measurement Sensor Information, and

3) Atmospheric and Tracer Measurements.

Each of these "logical" groupings (or "data stores") takes the form of a VAX/VMS/RMS keyed file in the actual implementation of the MEDb. Appendix $B$ contains a detailed description of the record structures of these files, including all record components, their data type, field length and range (where appropriate). The following section, MEDb Implementation Structure, identifies the corresponding equivalent file specification(s). The remainder of this section discusses each structure component in general terms The reader may find it useful to skim Appendix A at this point, and refer to Figure A-1 while reading the remainder of this MEDb Contents section.

(Note that the MEDb structure has been designed to store measurements as point $(x, y, z)$ data. Although initial use of this data archive will store field measurements, it can also be used to store results of analytical functions, if such results can be expressed as point data.)

\section{General Dataset Information:}

This "high-level" information contains the name of the dataset, map projection information used to convert latitude/longitude coordinates for all locations stored in the dataset, path names (i.e. file specifications) pointing to the dataset's physical media storage locations, and comments concerning the dataset as a whole. This information is contained in a single data record (one record per processed dataset) and is comprised of these components:

1) Dataset Name - a label given to the set of information described by this record, and the other two "high-level" category data records. The Dataset Name is also found in these other two sets of records and acts as the link between all "high-level" records associated with a given dataset. 
2) Central Ĺongitude, Central Latitude, Standard Parallels (2), Ellipsoid, and Datum - map projection information used in converting latitude and longitude coordinates to a specific dataset projection coordinate system. (All dataset locations are stored in both latitude/longitude coordinates and $X-Y$ coordinates for a'specific map projection.)

3) Map Projection - the name of the map projection used consistently for all dataset $X-Y$ locations.

4) Library Pathnames - four file specifications, each pointing to the physical media storage location of one of the dataset's Meteorological Station Library, Meteorological Sensor Library, Tracer Station Library, or Tracer Sensor Library. (See MEDb Implementation Structure section below.)

5) Comments - an area of limited size where textual information can be directly associated with this dataset.

6) Additional Information Pathname - a file specification pointing to the physical media storage location of additional comments associated with the dataset.

\section{Dataset Measurement Type Information:}

This information is typically comprised of a series of records, each record containing information for a single type of measurement found in the associated dataset. Some components are valid only for tracer measurement types (e.g. air concentrations, ground deposition, etc.), while others are valid for all types. Each record contains these components:

1) Dataset Name - the same label found in the General Dataset Information described above. As noted, this component is used to associate this record with its proper General Dataset Information record via this shared component.

2) Measurement Type - a label describing one of several kinds of data found in the associated dataset. The data may be either scalar (e.g. temperature) or vector (e.g. horizontal wind velocity) in nature.

3) Measurements Pathname - the file specification pointing to the physical media storage location containing the Atmospheric and Tracer Measurements data associated with this Measurement Type.

4) Start/Stop Times - the date and time of the first/last measurement (or beginning/ending date and time of the collection period of the first/last measurement) found in the Atmospheric and Tracer Measurements data file pointed to by the Pathname component. This provides for the flexibility to split relatively large amounts of a given dataset Measurement Type into multiple physical storage locations, allowing for access to only those data falling within a limited selected time period.

5) Units - a label indicating the measurement data units used when the data for this Measurement Type were stored in the Atmospheric and Tracer Measurements file. Note that this requires all data of a given Measurement Type to be stored in the same units for a given dataset. However Measurement Type units may vary from dataset to dataset. 
6) Minimum/Maximum Time Periods - the shortest/longest data collection period used in calculating a single stored measurement (value is non-zero if this Measurement Type is temporally averaged or integrated data).

7) Number of Measurements - the total number of dataset measurements for this Measurement Type recorded in the Atmospheric and Tracer Measurements file pointed to by the Pathname component.

8) Number of Measurement Stations - the number of different measurement stations at which data for this Measurement Type was taken. Different stations are typically defined by different latitude/longitude locations. However in some instances co-located instrumentation may constitute multiple stations (e.g. a three-level tower supposedly co-located with an airsonde release point would constitute two separate stations). See the Measurement Station Information subsection for more details.

9) Number of Measurement Sensors - the number of different measurement sensors (instruments) at all dataset measurement stations which recorded data for this Measurement Type. For example, a threelevel tower which records air temperature at each level would have three air temperature sensors for that station. The total number of sensors for all Measurement Types should equal the number of records found in the Measurement Sensor Information data file.

The following components are valid only for tracer concentration Measurement Types:

10) Material - the tracer compound (e.g. SF6, etc.) measured for this Measurement Type.

11) Minimum/Maximum Distance to Source - the closest/furthest measurement station from the tracer release location for this dataset Measurement Type.

12) Background Value - the ambient background tracer concentration associated with this Measurement Type.

13) Number of Background Measurements - the total number of dataset measurements for this Measurement Type having the Background Value which are recorded in the Atmospheric and Tracer Measurements file pointed to by the Pathname component.

\section{Dataset Source Term Information:}

Whereas other dataset structures, and their components, are somewhat entwined with each other, this information is relatively dissociated from other $\mathrm{MEDb}$ components. It is included primarily as an archive of the dataset's experimental tracer release source information to provide a convenient storage location for MEDb users. This information typically involves a series of records, one or more records for each separate dataset experiment involving the release of an atmospheric tracer material. The structure is comprised of these components:

1) Dataset Name - the same label as described above. 
2) Source Name - a secondary label indicating the specific experiment in the dataset with which this record information is to be associated.

3) Source Material - the tracer compound released.

4) Release Time On/Off - the dates and times when the tracer release began/ended.

5) Source Units - the units in which the tracer Amount Released is stored.

6) Amount Released - the amount of the tracer released expressed in the stored Source Units.

7) Source Latitude and Longitude - the latitude and longitude of the tracer release source location.

8) Source $X$ and $Y$ - the equivalent $X$ and $Y$ source location coordinates in the map projection indicated in the General Datàset Information.

9) Release Height - the height above the surface of the tracer release.

10) Terrain - the terrain elevation above sea level at the tracer release location.

11) Source Type - a categorization of the type of source for this experiment which describes the physical characteristics affecting the release (e.g. elevated, buoyant, passive, etc.).

12) Gas Temperature - the source gas emission temperature, for releases where thermal plume rise is significant.

13) Gas Velocity - the source gas exit velocity, for releases where momentum plume rise is significant.

\section{Measurement Station Information:}

This is the first of the three "lower-level" data structures. It contains information related to a specific data measurement station. Usually a station can be defined for this purpose as a unique measurement Latitude/Longitude location, however occasionally other station record components (e.g. Station Type or Level Type) or associated sensor record components (see Measurement Sensor Information subsection) may cause two or more stations to be defined at the same latitude/longitude. This structure is comprised of these components:

1) Station ID - a unique label given to a station to distinguish it from all other dataset stations. Much like the Dataset Name links the three "highlevel" data records, the Station ID acts as a link between records related to a given station in the three "lower-level" data structures.

2) Station Name - an extended, relatively more descriptive label associated with the station.-

3) Station Latitude and Longitude - the latitude and longitude of the station's location.

4) Station $X$ and $Y$ - the equivalent $X$ and $Y$ station location coordinates in the map projection indicated in the General Dataset Information.

5) Terrain - the terrain elevation above sea level at the station location.

6) Level Type - a categorization of the station indicating the number of its potential measurement levels (i.e. heights above the surface). 
7) Maximum Number of Levels - the maximum number of potential station measurement levels (if not "unlimited" as in an upper air. measurement station, e.g. data from a sodar, airsonde, etc.).

8) Station Type - a categorization of the station indicating whether the data collected is meteorological, tracer concentrations, or both.

9) Suspect Data Flag - a flag used to indicate some (usually negative) characteristic of the station which could affect data quality. The equivalent of this flag is present in all "lower-level" data structures, which affords the user an opportunity to associate this type of information at the station, sensor, or measurement level.

10) Data Use Flag - a flag used to indicate whether data from this station is to be used in subsequent processing for model evaluation purposes. The equivalent of this flag is present in all "lower-level" data structures, which affords the user an opportunity to associate this type of information at the station, sensor, or measurement level.

11) Comments - an area of limited size where textual information can be directly associated with this station.

\section{Measurement Sensor Information:}

This structure contains instrument-specific information. In this context, a "sensor" refers to the device used to acquire the measurement, which may record these measurements at one or more heights above the surface. A given station may have several associated sensors, one for each Measurement Type measured at each fixed height above the surface. However, some devices record multiple level data (e.g. sodar, airsonde, etc.). These devices will typically have one sensor record for each Measurement Type recorded by the device. This structure is comprised of these components:

1) Station ID - the same label found in the Measurement Station Information structure.

2) Measurement Type - the Measurement Type (see Dataset Measurement Type Information subsection) being recorded by the instrument.

3) Material - the tracer compound (if this is a tracer Measurement Type) being measured by the instrument.

4) Sensor Height - the height above the surface of the instrument taking the measurement (if taken at a fixed height).

5) Measurement Source - the type of instrument used to generate the measurements from this sensor, or other identifying data origin information.

6) Temporal Type - the type of temporal processing (e.g. instantaneous, averaged, integrated) used to generate the measurements from this instrument.

7) Time Period - the length of the time period over which data from this instrument are used to calculate the measurement (if Temporal Type is averaged or integrated).

8) Uncertainty - the magnitude of the uncertainty in the measurements taken by this instrument. 
9) Uncertainty Type - a flag indicating if the Uncertainty component is expressed as a percentage of the measurement or as a fixed constant. 10) Suspect Data Flag - the same component found in the Measurement Station Information structure, only applied to data from the sensor.

11) Data Use Flag - the same component found in the Measurement Station Information structure, only applied to data from the sensor.

\section{Atmospheric and Tracer Measurements:}

This structure holds the measurement data itself, and has a dual nature which depends on whether the measurements it holds are scalar or vector. Each Measurements data file is expected to contain data for only one Measurement Type as specified in the Dataset Measurement Type Information structure, and thus the type of data is not explicitly part of this structure. The structure's components are:

1) Station ID - the same label found in the Measurement Station and Sensor Information structures.

2) Measurement Time - the date and time of the measurement (if measurement is instantaneous), or the ending date and time of the measurement period (if measurement is averaged or integrated).

3) Measurement Height - the height above the surface of the measurement.

4) Measurement - the scalar measurement value,

or

4) Measurements - the two measurements which together form the vector measurement.

5) Suspect Data Flag - the same component found in the Measurement Station and Sensor Information structures, only applied to the single measurement record.

6) Data Use Flag - the same component found in the Measurement Station and Sensor Information structures, only applied to the single measurement record.

\section{MEDb Implementation Structure}

As mentioned earlier, each of the data structures listed in the previous section have been implemented as keyed records in VAX/VMS/RMS MEDb files. It is first useful to describe the overall MEDb file storage directory structure. Figure 2 illustrates the storage location of the various database files. For purposes of illustration in this document, all MEDb files are found under the rooted logical "dsk\$modev" (which is equivalent to "dsk\$modev_root:[datasets.]" where dsk\$modev_root is a defined system logical). The top-level directory "dsk\$modev:[000000]" contains various sub-directories, each one (except for "all_sets.dir") containing the MEDb files for a particular dataset.

Each of these dataset sub-directories in turn contain three other directories. These directories are dsk\$modev:[(dataset ID).raw], [(dataset ID).code], and [(dataset ID).formatted], where (dataset ID) is a shortened form of the Dataset Name. The [.raw] directories contain the unprocessed raw data files 
for that dataset. The [.code] directories typically contain the Pascal source code used in processing the raw data files into the formatted MEDb files. These formatted MEDb files are stored in the [.formatted] directories. Each directory also typically contains an "aareadme.txt" file describing the files in the directory. The [.formatted]aareadme.txt files are most descriptive and contain detailed information concerning the measurements and processing of the data.

The "dsk\$modev:[all_sets]" sub-directory is a special case. It contains the files holding the MEDb "high-level" data structures, which are found in [all_sets.formatted]. The code which created these files is in [all_sets.code]. The [all_sets.raw] directory is empty.

Returning now to the structures described in the MEDb Contents section, the file dsk\$modev:[all_sets.formatted]dataset_list.dat contains the equivalent of the General Dataset Information. The records (at least one for each unique Measurement Type - Material combination) holding the Dataset Measurement Type Information are in [all_sets.formatted]measurements_in_datasets.dat. And the file [all_sets.formatted]sources_in_datasets.dat contains the equivalent of the Dataset Source Term Information.

The remaining MEDb files corresponding to the "lower-level" data structures for each processed dataset are stored in their respective [.formatted] directory. The Measurement Station Information is, in practice, split into two files. One contains information for all dataset tracer stations (station_library.dat), while the other contains records for all meteorological station information (station_library_met.dat). Note that this is not a requirement, in that both Station Pathnames in the General Dataset Information could point to the same file. This is done simply to limit the number of records required to be processed, assuming users will wish to access either tracer stations or meteorological stations at a given time. For similar reasons, the Measurement Sensor Information is split into two files. One for all tracer sensors (sensor_library.dat), and one for meteorological sensors (sensor_library_met.dat).

Several files typically contain the Atmospheric and Tracer Measurements data for a given dataset. Each file will contain measurements for a single Measurement Type, and will contain that Type as the first part of its filename. The complete list of these Types is given in Appendix B. The filename may be modified to also include the Material (if the file contains a tracer Measurement Type) and/or a 6-character date (yymmdd) if the Measurement Type data has been split into several files by UTC measurement date. This is sometimes done for large datasets containing 10,000+ measurements for a given Measurement Type. In practice, the Material is added to the filename only if more than one Material is used as a tracer for a given dataset. Examples of actual measurements filenames are:

AvgAirCcn.dat

AirTemp_910223.dat
AvgAirCcn_SF6.dat

WndSpdDir_910208.dat
AirTemp.dat

WndSpdDir.dat The above structures allow flexibility in determining how the MEDb data and library information for a given dataset will be split into files. 


\section{MEDb Data Issues}

This section contains information related to the filling of the MEDb with data from the first few datasets that have been processed. Users should be aware that the MEDb entries for these datasets are incomplete. Every record component has not yet been filled, and every possible dataset Measurement Type has not always been processed.

Unfilled record fields or fields which are not applicable (e.g. the Sensor Height for a tethersonde station in the Measurement Sensor Information) will always be blank-filled for "character string" fields, contain -99999 for "integer" fields, and contain -99999.0 for "real" fields (refer to Appendix B). Those fields which may be applicable, but are as of now usually unfilled, are:

1) Minimum/Maximum Distance to Source (Dataset Measurement Type Information)

2) Some Terrain fields where the information has not yet been found

(Dataset Source Term and Measurement Station. Information)

3) Source Type (Dataset Source Term Information)

4) Uncertainty (Measurement Sensor Information)

As time permits, or as the need for this information becomes more important, these fields will be completed, where possible.

Most available measurement data has been processed. However some (e.g. cloud observations, visibility, etc.) still remains only in raw form. A list of the unprocessed data can be found in the [(dataset ID).formatted] aareadme.txt files for each dataset. These files are typically those pointed to by the Additional Information Pathway component of the General Dataset Information record. Users of a particular dataset are STRONGLY urged to read these files as they contain important dataset-specific information concerning processing methods and other key issues. (In the case of any conflicting information between the aareadme.txt files and this document, the user should typically be guided by the aareadme.txt files which will likely be more current.)

$\mathrm{Be}$ aware that no MEDb documentation is meant to replace the much more detailed published experiment reports. These reports, when available, are filed with hard copies of each dataset's MEDb Station and Sensor Libraries, Dataset Measurement Type records, Dataset Source Term records, and Additional Information files. The user should refer to these documents for detailed information concerning the experiment, and for an overview of the dataset's $\mathrm{MEDb}$ contents.

Users are warned that although some simple individual measurement range checking has been done during processing of the raw data, $M E D b$ Atmospheric and Tracer Measurements remain largely unchecked for data value consistency and quality (beyond that indicated in the dataset's Additional Information file). If, for example, atmospheric pressure data were to increase with height above the surface, this would not be noted during processing. Users must review any data they use for apparent quality.

The MEDb is meant to be an evolving database where users supply updated information concerning the quality and usefulness of the data through the use of the Comments, Suspect Data Flags, Data Use Flags, and other fields. 
Users will be able to access and modify their own copy of the master MEDb (see the accompanying RAS Division Model Evaluation Database Tools document). Users should then refer any permanent MEDb changes to the MEDb coordinator. As users make modifications to the MEDb they should be aware of certain conventions which have been used to date. These are:

1) Meteorological Station IDs have typically be kept to eight characters or less. Although the MEDb field length is ten characters, the MEDIC model input station length limit is eight characters. If the MEDb stations are used as input to the Format_MEDIC_Input code (refer to the "Format MEDIC Input" document), the first eight characters of any meteorological Station ID must be unique (the first seven must be unique if the station is a fixed-level tower reporting winds at more than one level). Meteorological Station IDs usually follow the convention of using up to the first four characters to meaningfully distinguish the station from others, then after a blank use the following three character codes:
AIR for aircraft measurements
PRF for profilers
SDR for sodars
SND for all types of sondes
TWR for tower stations

Examples are:
A TWR
ARV TWR
FOOT TWR
BR SDR
CSL AIR

2) Measurement Types have been named in an attempt to convey the maximum amount of information in ten characters. All wind related Types begin with the substring "Wnd". The first character of each substring representing a word is capitalized (e.g. AirTemp for air temperatures). Refer to Appendix B for a list of all Types used to date. Measurement Types were entered into the Dataset Measurement Type Information file in alphabetical order to facilitate their ordered display when examining these records (see "RAS Division Model Evaluation Database Tools" document).

3) When data for a particular dataset Measurement Type has been split into multiple files, a record for each of the files will appear in the Dataset Measurement Type Information file. Only the Measurements Pathname, Start/Stop Times, Number of Measurements, and Number of Background Measurements fields have values pertaining to the individual files. The remaining fields have values for all files of the given Measurement Type series taken together. For example, if a Minimum Time Period entry is 900 (15 minutes) for one of several AirTemp files, this means that 900 seconds is the shortest averaging period for some air temperature data in one of the files, and not necessarily in the one file pointed to by that record's Measurements Pathname. Also, in these multiple Type file cases, since all files have been split to contain all data based on UTC date, the Start Time associated with any file but the first Measurements file in the series is 
assigned a time of 00:00:00 (hh:mm:ss) and the Stop Time associated with any file but the last file in the series is assigned a time of 23:59:59. This is regardless of the actual first and last Measurement Time found in these Measurement files. All other Start and Stop times in the Dataset Measurement Type Information records do reflect the actual times of the first and last records in the associated Atmospheric and Tracer Measurements file. 


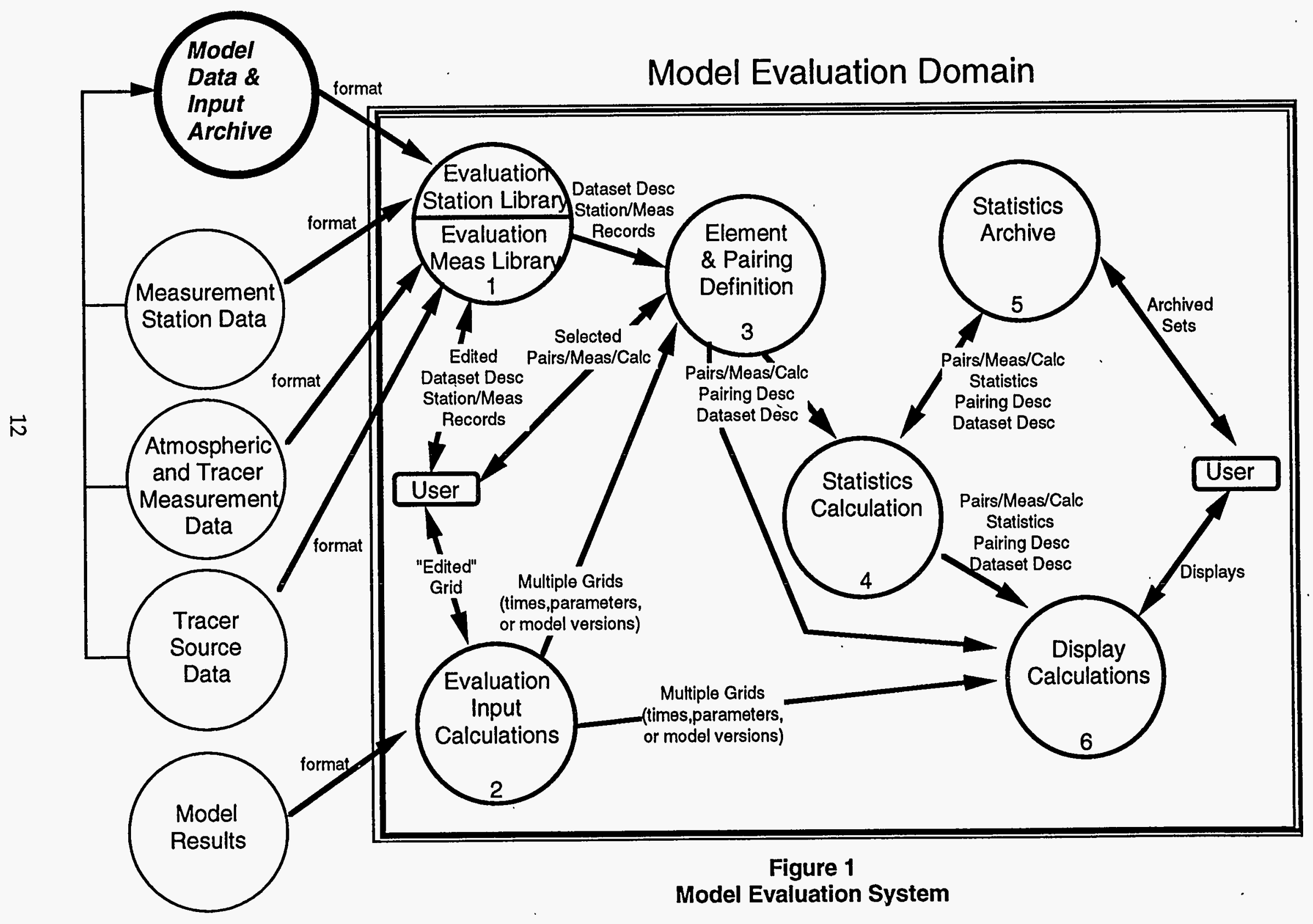




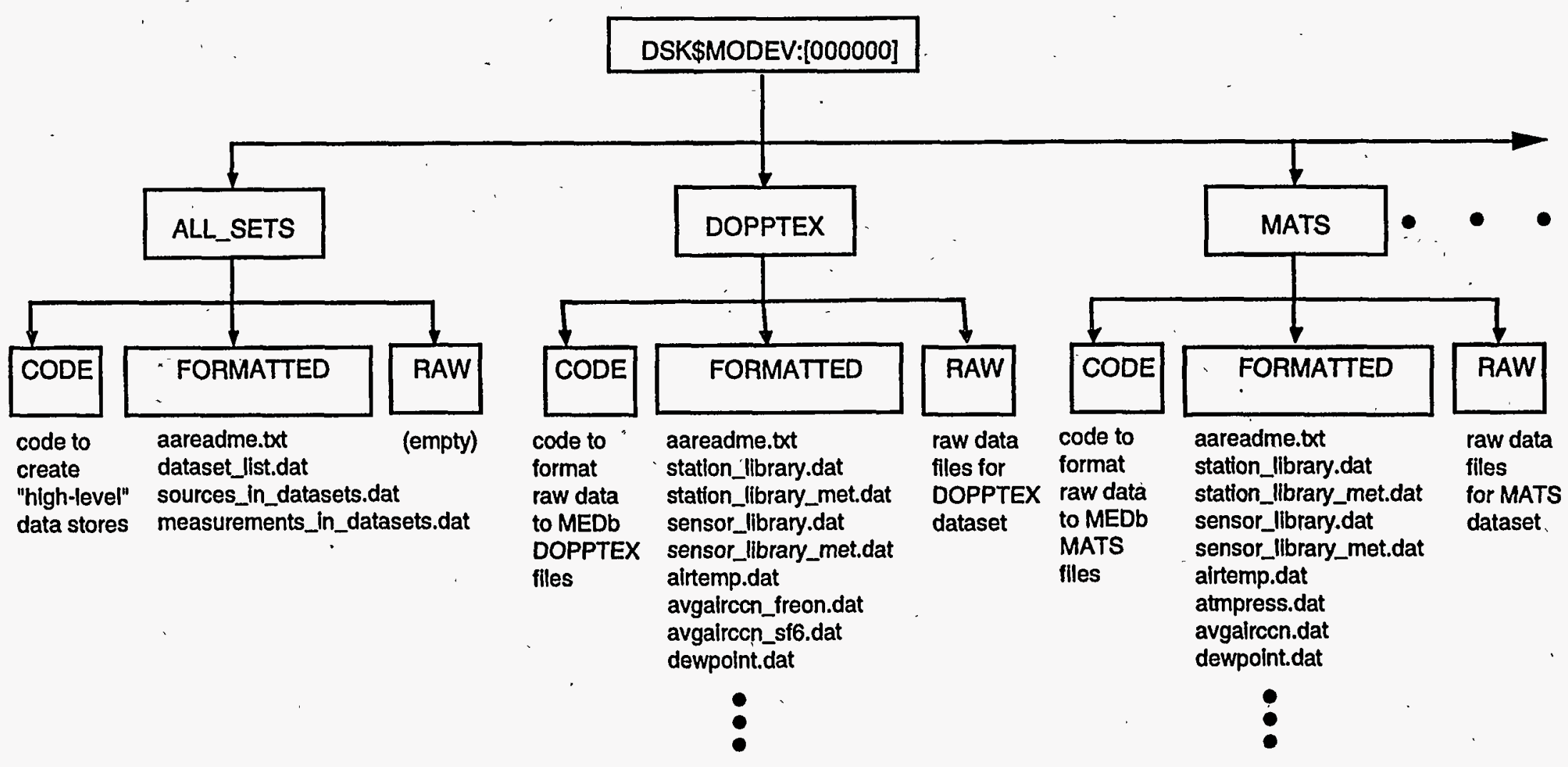

Figure 2

MEDb Directory Structure 


\section{APPENDIX A \\ MEDb Configuration}

Figure A-1 is a diagram showing the components (fields) of each MEDb storage structure (record), along with the logical data store names and their equivalent implemented file specifications. Note that the components are listed in order of discussion in the MEDb Contents section, which differs from the actual field order in the physical records listed in Appendix B. As the diagram key illustrates, the logical data store name is shown above the rectangle containing the data store components for each data structure discussed in the main text of this document. The equivalent file specification is listed below each rectangle.

Dashed arrows highlight fields which act as logical links between the various structures. (Two dash types are used. This is merely to distinguish one arrow from another where the arrows cross.) These links are:

1) Dataset Name - which is present in all three "High-Level" data structures. This serves to identify all General, Measurement Type, and Source Term Information records for a particular dataset.

2) Station ID - which is present in all three "Lower-Level" data structures.

- This serves to identify all Station, Sensor, and Measurement records for a particular measurement station.

3) Library Pathnames (4) - present in the General Dataset Information which identifies the four Station and Sensor Library files associated with each dataset:

4) Measurements Pathname - present in the Dataset Measurement Type Information which identifies the file containing dataset measurements of a given type.

5) Measurement/Sensor links - which tie a given measurement to the sensor which acquired the measurement. In addition to the Station ID, these are the Measurement/Sensor Heights found in the Measurements and Sensor Information data structures, along with the Measurement Type and Material listed in the Dataset Measurement Type Information record pointing to the Atmospheric and Tracer Measurements record in which the measurement is found. This combination of the Station ID and Measurement Height found in the Measurements record, along with the Measurement Type and Material represented by the accessed Measurements file, defines a unique Sensor Information record. With these links, all relevant MEDb records can be identified for a particular measurement from other fields in the measurement record and by knowing the file specification of the file containing that measurement. 
"High-Level" Data Stores

General Dataset Information

\begin{tabular}{|l|}
\hline Dataset Name \\
Central Longitude \\
Central Latitude \\
Standard Parallel 1 \\
Standard Parallel 2 \\
Ellipsoid \\
Datum \\
Map Projection \\
Meteorological Station Library Pathname \\
Tracer Station Library Pathname \\
Meteorological Sensor Library Pathname \\
Tracer Sensor Library Pathname \\
Comments \\
Additional Information Pathname \\
dsk\$modev:[all_sets.formatted] \\
$\quad$ dataset_list.dat
\end{tabular}

Dataset Name

Central Longitude

Central Latiude

Standard Parallel 1

Standard Parallel 2

Ellipsoid

Meteorological Station Library Pathname

Tracer Station Library Pathname

Meteorological Sensor Lbrary Pathname

Tracer Sensor Library Pathname

Comments

dsk\$modev:[all_sets.formatted] dataset_list.dat

1 Dataset Measurement Type Information

I

Dataset Name

Measurement Type

Measurements Pathname

Start Time

Stop Time

Units

Minimum Time Period

Maximum Time Period

Number of Measurements

Number of Measurement Stations

Number of Measurement Sensors

Material

Minimum Distance to Source

Maximum Distance to Source

Background Value

Number of Background Measurements

dsk\$modev:[all_sets.formatted]

measurements_in_datasets.dat

1 Dataset Source Term Information

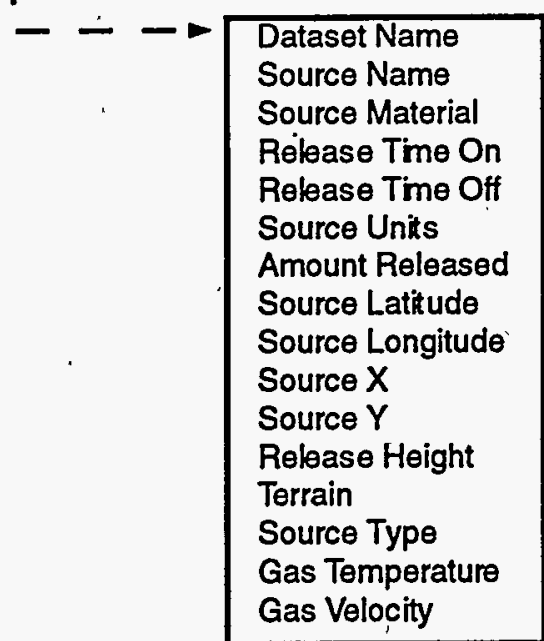

dsk\$modev:[all_sets.formatted]

sources_in_datasets.dat
"Lower-Level" Data Stores

Measurement Station Information

\begin{tabular}{|c|c|}
\hline $\begin{array}{l}\text { Station ID } \\
\text { Station Name } \\
\text { Station Latitude } \\
\text { Station Longkide } \\
\text { Station X } \\
\text { Station Y } \\
\text { Terrain } \\
\text { Level Type } \\
\text { Max. Nbr. of Levels } \\
\text { Station Type } \\
\text { Suspect Data Flag } \\
\text { Data Use Flag } \\
\text { Comments }\end{array}$ & $\begin{array}{l}\text { Station ID } \\
\text { Station Name } \\
\text { Station Latitude } \\
\text { Station Longitude } \\
\text { Station X } \\
\text { Station Y } \\
\text { Terrain } \\
\text { Level Type } \\
\text { Max. Nbr. of Levels } \\
\text { Station Type } \\
\text { Suspect Data Flag } \\
\text { Data Use Flag } \\
\text { Comments }\end{array}$ \\
\hline
\end{tabular}

dsk\$modev:[(dataset ID).formatted]

station_library.dat $\rightarrow$ station_library_met.dat (tracer library) 」 (meteorological library)

Measurement Sensor Information

Station ID

Measurement Type

Material

Sensor Height

Measurement Source

Temporal Type

Time Period

Uncertainty

Uncertainty Type

Suspect Data Flag

Data Use Flag

Station ID

Measurement Type

Material

Sensor Height

Measurement Source

Temporal Type

Time Period

Uncertainty

Uncertainty Type

Suspect Data Flag

Data Use Flag

dsk\$modev:[(dataset ID).formatted]

sensor_library.dat $\rightarrow$ sensor_library_met.dat (tracer library)

(meteorological library)

Atmospheric and Tracer Measurements

Station ID

Measurement Time Measurement Time

- Measurement Height Measurement Height

Measurement

Suspect Data Flag

Data Use Flag

Measurement (Wind Speed)

Measurement (Wind Direction)

Suspect Data Flag

Data Use Flag

I

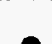

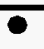

1

I

1 dsk\$modev:[(dataset ID).formatted]

$--\ldots$ (measurement_type)_(modifier).dat

\begin{tabular}{|cc|}
\hline Date Store Key: & Logical Name \\
& Components \\
data links & File Name \\
\hline & \\
\hline
\end{tabular}




\section{APPENDIX B \\ MEDb Record Definitions}

The following lists the record structure used for each MEDb RMS record. The variables are listed in the order they are written in their respective records. The variable name is followed by a description of the variable's data type and, where appropriate, notes concerning valid values and units. All integers are 32bit signed variables. All reals are 32-bit single precision variables. ("*" indicates a keyed field.)

\section{Dataset_List.Dat: \\ Variable \\ Dataset Name* \\ Central Longitude \\ Central Latitude \\ Standard Parallel 1 Standard Parallel 2 Map Projection}

Ellipsoid

Datum

Comments

Met Sensor Library Path Met Station Library Path Tracer Sensor Lib Path Tracer Station Lib Path Additional Info Path

\section{Description}

50 Character String.

Real. Positive implies Eastern hemisphere. Negative implies Western hemisphere.

Real. Positive implies Northern hemisphere.

Negative implies Southern hemisphere.

Real.

Real.

32 Character String. Currently all Map Projections are Universal Transverse Mercator.

32 Character String. Currently all Ellipsoids are Clarke 1866.

32 Character String. Not currently used.

1000 Character String. Any printable character.

Strings usually limited to relatively short, important messages concerning the use of the dataset.

64 Character String. Valid RMS file specification.

64 Character String. Valid RMS file specification. 64 Character String. Valid RMS file specification. 64 Character String. Valid RMS file specification. 64 Character String. Valid RMS file specification.

Measurements_In_Datasets.Dat:

Variable

Dataset Name* Measurement Type
Description

50 Character String.

10 Character String. String describes a type of data which has been measured or has been derived from measurements. Types currently in use:
AirTemp
- sensible air temperatures
AtmPress - atmospheric pressures
AvgAirCcn - averaged air concentrations (tracer)
DewPoint - dew point temperatures 
Start Time

Stop Time

Units

Minimum Time Period Maximum Time Period Number Measurements Number of Meas Stations Number of Meas Sensors Measurements Pathname Material

Minimum Dist to Source

Maximum Dist to Source

Background Value

Nbr of Background Meas
HorzNetRad - net radiation on a horizontal plane at the surface

HorzSolRad - solar radiation on a horizontal plane at the surface

Inversions - base of thermal inversion layer

MixingHgt -atmospheric mixed layer depth (derived)

StabClass - atmospheric stability class (derived)

WndSigPhi - standard deviation of the wind elevation angles

WndSigSpd - standard deviation of horizontal wind speeds

WndSigSpdW- standard deviation of vertical wind speeds

WndSigThe - standard deviation of the wind directions

W.ndSpdDir - horizontal wind speeds and directions (vector)

WndSpdW - vertical wind speeds

12 Character String (yymmddhhmmss) in UTC.

12 Character String (yymmddhhmmss) in UTC.

10 Character String. Units currently in use:

Concentrations - PPT, ng/m3

Directions - deg

Heights - meters

Pressures - millibars

Radiation - watts/m2

Speeds $\quad-m / s$

Temperatures - Celsius

Wind velocity vector $-\mathrm{m} / \mathrm{s}$ deg

Integer. Seconds.

Integer. Seconds.

Integer.

Integer.

Integer.

64 Character String. Valid RMS file specification.

10 Character String. For tracer Measurement Types

only. Materials currently in use: Freon-F3B1, SF6

Real. Kilometers. For tracer Measurement Types only.

Real. Kilometers. For tracer Measurement Types only.

Real. In units consistent with Units field listed above.

For tracer Measurement Types only.

Integer. For tracer Measurement Types only. 
Sources_In_Datasets.Dat:

Variable

Dataset Name*

Source Name

Source Material

Release Time On

Release Time Off

Source Units

Amount Released

Source $X$

Source $Y$

Release Height

Source Latitude

Source Longitude

Terrain

Source Type

Gas Temperature

Gas Velocity
Description

50 Character String.

50 Character String.

10 Character String. Materials currently in use:

Freon-F3B1, SF6

12 Character String (yymmddhhmmss) in UTC.

12 Character String (yymmddhhmmss) in UTC.

10 Character String. Units currently in use:

$\mathrm{g} / \mathrm{sec}, \mathrm{Kg} / \mathrm{hr}$

Real. Consistent with Source Units field listed above

Real. Kilometers.

Real. Kilometers.

Real. Meters.

Real. Decimal degrees. Positive implies Northern hemisphere. Negative implies Southern hemisphere.

Real. Decimal degrees. Positive implies Eastern hemisphere. Negative implies Western hemisphere. Integer. Meters.

10 Character String. Not yet used.

Real. Celsius.

Real. Meters/Second.

Station_Library(Met).Dat:

Variable

Station ID*

Station Name

Station $X$

Station $Y$

Station Latitude

Station Longitude

Terrain

Maximum $\mathrm{Nbr}$ of Levels

Level Type

Station Type
Description

10 Character String. Note: if this station is to be used for MATHEW/ADPIC meteorological data input, suggest limiting Station ID to 8 characters ( 7 is even better for multi-level towers) to be consistent with MEDIC station string length.

25 Character String.

Real. Kilometers.

Real. Kilometers.

Real. Decimal degrees. Positive implies Northern hemisphere. Negative implies Southern hemisphere. Real. Decimal degrees. Positive implies Eastern hemisphere. Negative implies Western hemisphere. Integer. Meters.

Integer. For Level Types of "S" or "F".

Character. One of:

$\mathrm{S}$ Single measurement height

F Fixed (multi-level) measurement heights

U Unlimited or variable measurement heights Character. One of:

M Meteorological measurements

$\mathrm{T} \quad$ Tracer concentration measurements 
Suspect Data Flag

Data Use Flag

Comments
B Both types of measurements

Character. Flags currently in use:

L Station location is approximate

Character. One of:

$Y$ Use

$\mathrm{N}$ Don't use

1000 Character String.

Sensor_Library(_Met).Dat:

Variable

Station ID*

Sensor Height

Measurement Type

Material

Measurement Source

Temporal Type

Time Period

Uncertainty

Uncertainty Type

Suspect Data Flag

Data Use Flag
Description

10 Character String. (See Station ID note above.)

Real. Meters.

10 Character String. (See Measurement Type above for list.)

10 Character String. (See Material above for list.)

10 Character String.

3 Character String. One of:

AVG Averaged

INS Instantaneous

INT Integrated

Integer. Seconds.

Real.

Character. One of:

F Uncertainty value is a fixed constant for all sensor measurements

$\% \quad$ Uncertainty value is expressed as a percentage of the measurements

Character. None yet used for sensors

Character.

Y Use

N Don't use

Measurements Files (scalar):

Variable

Measurement Time*

Station ID*

Measurement Height

Measurement

Suspect Data Flag
Description

12 Charàcter String (yymmddhhmm) in UTC.

10 Character String. (See Station ID note above.)

Real. Meters.

Real. In units consistent with Units field found in Measurements_In_Datasets.Dat record.

Character. One of:

A Data averaging period significantly differs from that given in Measurement Sensor Information record.

D Data was recorded at the same time, location and height as a previously entered measurement (occurs with tethersondes). 
$\mathrm{R} \quad$ Measurement exceeds a more-than-reasonable range (or Measurement Height is less than zero). These ranges consistent with ARAC constraints database.

Q SODAR data quality judged to be poor due to a low data reliability calculation.

Data Use Flag

$$
\begin{aligned}
& \text { Data has some unspecified factor(s) affecting } \\
& \text { measurement quality }
\end{aligned}
$$

Character.

Y Use

$\mathrm{N}$ Don't use

Measurements Files (vector) (note that to date all vector files contain winds):

Variable

Description

Measurement Time*

12 Character String (yymmddhhmm) in UTC.

Station ID*

Measurement Height 10 Character String. (See Station ID note above.)

Wind Speed

Wind Direction

Real. Meters.

Real. In units consistent with Units field found in Measurements_In_Datasets.Dat record.

Real. In units consistent with Units field found in Measurements_In_Datasets.Dat record.

Suspect Data Flag

Character. One of:

A Data averaging period significantly differs from that given in Measurement Sensor Information record.

D Data was recorded at the same time, location and height as a previously entered measurement (occurs with tethersondes).

M One of the vector measurements is missing.

$\mathrm{R} \quad$ At least one of the vector measurements exceeds a more-than-reasonable range (or Measurement Height is less than zero). These ranges consistent with ARAC constraints database.

Q SODAR data quality judged to be poor due to a low data reliability calculation.

? Data has some unspecified factor(s) affecting measurement quality

Data Use Flag

Character.

Y U Use

$\mathrm{N}$ Don't use 